\title{
ESTIMATING RANDOM POLYNOMIALS BY MEANS OF METRIC ENTROPY METHODS
}

\author{
MICHEL WEBER
}

\begin{abstract}
The purpose of this paper is to indicate how easy, classical metric entropy methods arising from the theory of stochastic processes, apply to get uniform estimates for random polynomials like the well-known Salem-Zygmund's bound [7]. As an application, we give a criterion for uniform convergence of some random Fourier series.
\end{abstract} test.

Mathematics subject classification (1991): 60F99, 28D99.

Key words and phrases: random polynomials, metric entropy, covering numbers, Dudley's integral

\section{REFERENCES}

[D] DUDLEY R. M., The size of compact subsets of Hilbert space and continuity of Gaussian processes, J. Functional Analysis 1 (1967), 290-330.

[F] FERNIQUe X., Un exemple illustrant l'emploi des méthodes gaussiennes, Actes de la "Conférence en l'honneur de J.P. Kahane", (Orsay, 1993). J. Fourier Anal. Appl., Special Issue (1995), 209-215.

[G] GaRsia A., Topics in almost sure convergence, Lectures in Adv. Math. Markham Pub. Company., 1970.

[GPW] Graversen, S.E., Peskir G., Weber M., The continuity principle in exponential type Orlicz spaces, Nagoya Math. J. 137 (1995), 55-75.

[K] Kahane J. P., Some Random Series of Functions, first edition, D.C Heath and Company, Second edition, Cambridge University Press, 1968.

[KS] Kashin B. S., SAakyan A. A., Orthogonal series, Translation of Math. Monographs 75, Amer. Math. Series (1989).

[KLS] Klein A., LANDAu L. J., ShUCKeR D. S., Decoupling inequalities for stationary Gaussian processes, Ann. of Prob. 10 (1982), 702-708.

[LT] Ledoux M., TAlagrand M., Probability in Banach spaces, Springer Verlag, 1990.

[PSW] PESKIR G., SCHNEIDER D., Weber M., Randomly weighted series of contractions in Hilbert spaces, Math. Scand. 79 (1996), 263-282.

[SW] SCHNEIDER D., WEBER M., Weighted averages of contractions along subsequences, Proceedings of the "Conference on convergence in ergodic theory and probability", Columbus June 1993, Ohio, W. de Gruyter and Co., Berlin-New-York, 1996, pp. 399-404.

[SZ] SAlEm R., ZYGMund A., Some properties of trigonometric series whose terms have random signs, Acta Math. 91 (1954), 245-301. 